\title{
ON THE AGGREGATION OF INDIVIDUAL PRIORITIES IN INCOMPLETE HIERARCHIES
}

\author{
Daniel Kunzler de Souza Carmo \\ Production Department \\ Sao Paulo State University \\ Guaratingueta, SP, Brazil \\ Email:daniel@dkcarmo.com \\ Fernando Augusto Silva Marins \\ Production Department \\ Sao Paulo State University \\ Guaratingueta, SP, Brazil \\ Email: fmarins@feg.unesp.br \\ Valerio A. P. Salomon* \\ Production Department \\ Sao Paulo State University \\ Guaratingueta, SP, Brazil \\ Email: salomon@feg.unesp.br \\ Carlos Henrique Pereira Mello \\ Institute of Industrial Engineering and Management \\ Federal University of Itajuba \\ Itajuba, MG, Brazil \\ Email: carlos.mello@unifei.edu.br
}

\begin{abstract}
One way to conduct group decision-making in AHP is asking judgments from different experts. The following step is the aggregation of these judgments. However, at first, it is important to note if experts share common values. That is, if they work for the same company or department, for instance. In this case, the aggregation of individual judgments (shorted as AIJ) by their geometrical mean seems to be proper. If the experts came from different organizations, they may not act as unit: then, the indication is the aggregation of individual priorities (AIP). According to the literature, to AIP, one may either take geometric mean or arithmetic mean of the resulting priorities. This work addresses the AIP of incomplete hierarchies. An incomplete hierarchy is a structure when not all elements of a level connect to all elements from the higher level. A hierarchy with goal, criteria and sub-criteria and without alternatives is an example of incomplete hierarchy. A decision model without alternatives seems to be unusual. Nevertheless, this kind of situation is very common, in practice. There is a large number of AHP applications with incomplete hierarchies, including prioritization of customer needs, specification of product requirements, identification of weights for quality service evaluation, to name a few. This work shows that for incomplete hierarchies, the arithmetic mean must be used to AIP. That is, using the geometric mean, the priorities for the lower level elements will sum different from the priority of the higher element. A mathematical modeling of this observation is presented and illustrated with a simple numeric example with two criteria and four sub-criteria.
\end{abstract}

Keywords: aggregation of individual priorities; group decision-making; incomplete hierarchy.

${ }^{*}$ Corresponding author 


\section{Introduction}

Analytic Hierarchy Process (AHP) is a multiple criteria decision-making (MCDM) method, widely applied by practitioners and researchers to prioritize. An overview of its applications comprises competitive benchmarking, market analysis, product development, and resource allocation, to name a few (Vaidya \& Kumar, 2006). AHP basis is the "innate human ability to use information and experience to estimate relative magnitudes through paired comparisons" (Saaty, 2006). The overall priorities of the hierarchy elements are then calculated using AHP procedure to help decision-makers to choose among alternatives.

Several situations require gathering assessments from a group of individuals to compose the final prioritization. For instance, all scenarios that a more than one decision-maker (customers, users, experts, stakeholders, and others) is present require a group decision method. Using AHP method, in order to aggregate their opinions there are several possible ways, including: (1) aggregating the individual judgments (AIJ) for each set of pairwise comparisons into an aggregate hierarchy; (2) synthesizing each of the individual's hierarchies and aggregating the resulting priorities (AIP - aggregating individual priorities); and (3) aggregating the individual's derived priorities in each node in the hierarchy. In any case, the relative importance of the decision-makers may either be assumed to be equal, or else incorporated in the aggregation process (Forman \& Peniwati, 1998). The two most common methods for group decision are AIJ and AIP. In first one, a judgment matrix is constructed for the group using geometric mean of all individual judgments and global and local priorities are calculated using the AHP procedure. In AIP, local priorities of each individual are first calculated and group priorities are obtained using geometric or arithmetic mean (Altuzarra et. al., 2007).

Hierarchy plays an important role on AHP and it must be defined by the application being studied. As illustrated on Figure 2, "a hierarchy does not need to be complete, that is, an element in a given level does not have to function as an attribute (or criterion) for all the elements in the level below" (Saaty, 1990). When such structure occurs, a hierarchy is called "incomplete" and it "can be divided into sub hierarchies sharing only a common topmost element" (Saaty, 2006). While most AHP applications have complete hierarchies (often a goal-criteria-alternative framework), important exceptions, can be seen on several situations, as product development, for instance (Armacost et al., 1994; Kwong \& Bai, 2002; Aykroyd, 2008).

This work shows that for incomplete hierarchies, the arithmetic mean must be used to AIP. That is, using the geometric mean, the priorities for the lower level elements will sum different from the priority of the higher element. We present a mathematical modeling of this observation and illustrate with a simple numeric example with two criteria and four sub-criteria. 


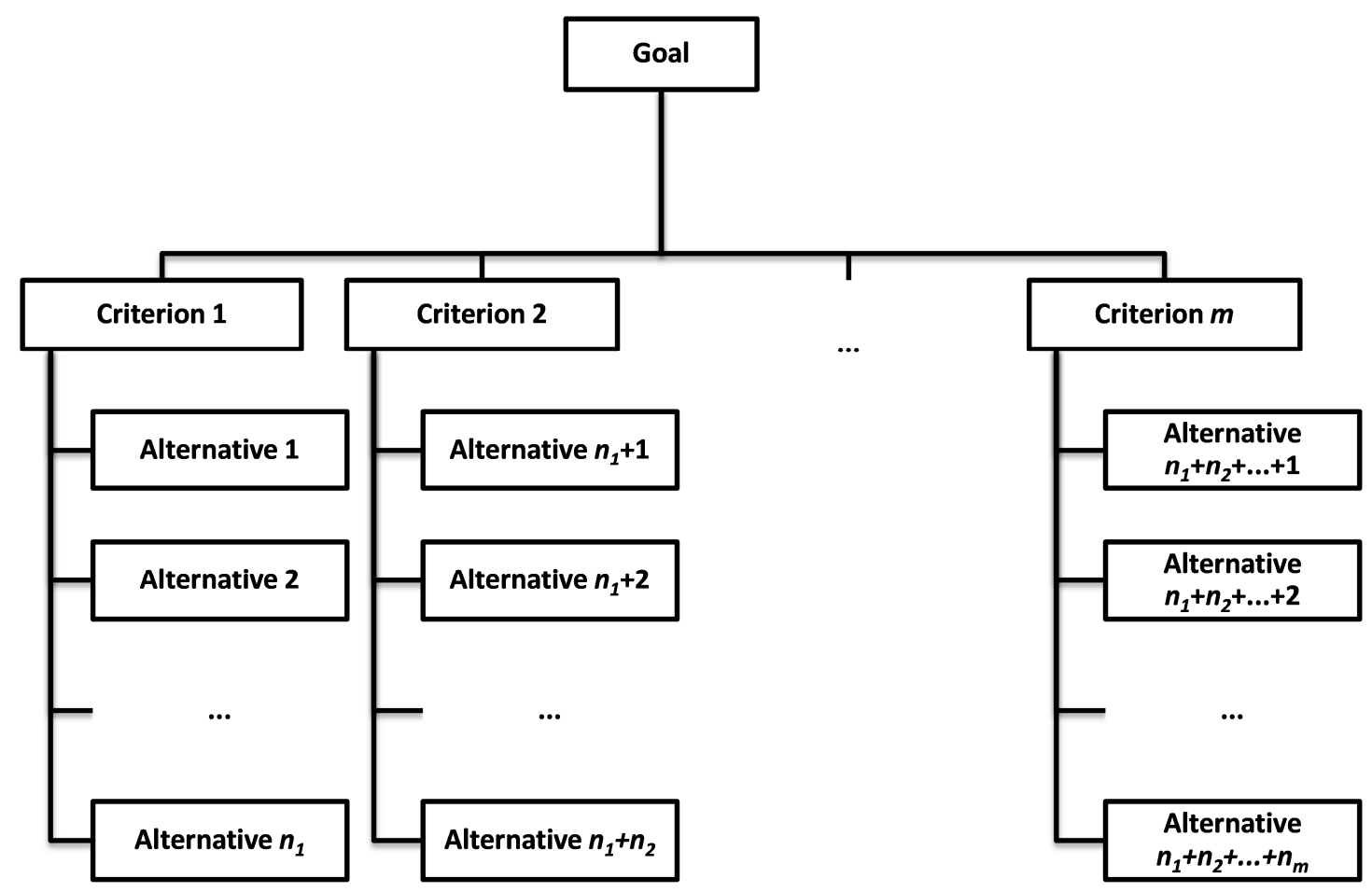

Figure 1. Incomplete hierarchy with alternatives contributing to a single criterion (Salgado et. al., 2012).

\section{Theoretical background}

When several individuals provide judgments to AHP, depending if the group assumed to act together as a unit, or as separated individuals, these judgments can be directly aggregated (AIJ), or else, just the aggregation of their resulting priorities (AIP) make sense. If the group shares common values and goals, usually composing the same organization, AIJ by the geometric mean should be used, satisfying the reciprocity principle, "implying a synergistic aggregation of individual preferences in such a way the group becomes a new 'individual' and behaves like one" (Forman \& Peniwati, 1998). Otherwise, if the group is a set of individuals, usually belonging to different organizations, with different agendas, "one may take either a geometric mean or an arithmetic mean of their resulting priorities".

AHP has been used for many applications and situations to help decision-makers to choose and prioritize alternatives in a hierarchy of criteria or sub-criteria or sub-objectives or categories. These hierarchies are comprised of at least two levels, with alternatives and a goal as their basic elements (bottom and top levels, respectively), but usually, AHP applications have criteria and sub-criteria levels between them, because it is an MCDM method. On applications with more than two levels, hierarchy can be a complete or incomplete arrangement of elements.

A natural way to organize an AHP application is with a complete hierarchy, as illustrated in Figure 2. All alternatives address against all criteria and sub-criteria. This is very common to the overall prioritization in MCDM. 


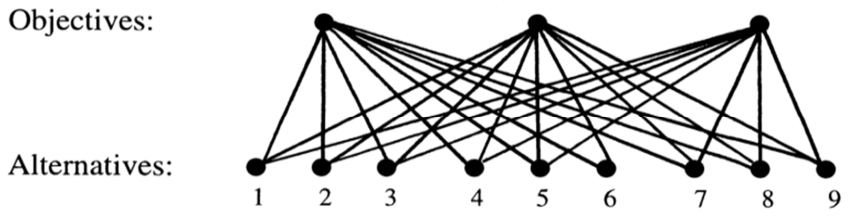

Figure 2. Complete hierarchy (Davies, 2001).

However, for some AHP applications, a complete hierarchy does not make sense. In Product development, for instance, customer needs may be organized into logical groups, also known as called "categories" (Kwong \& Bai, 2002). Every group shall be formed by exclusive elements, also named as "attributes". This is a major case of incomplete hierarchy, as the presented in Figure 3.

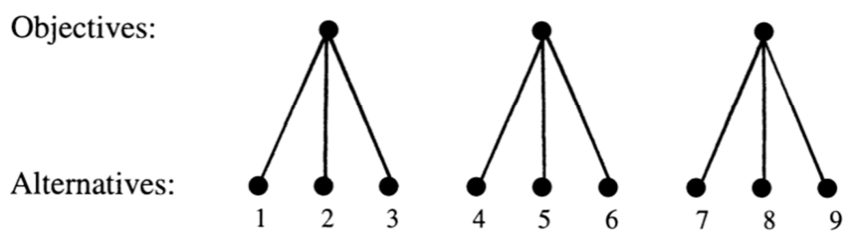

Figure 3. Hierarchy for a house selection (Davies, 2001)

The clustering procedure of incomplete hierarchies avoids possible errors, once "comparisons of elements in pairs requires that they be homogeneous or close with respect to the common attribute" (Saaty, 1990). Several publications show this procedure: Chan \& Wu (2005) propose grouping customer needs by Affinity Diagram or Cluster Analysis for product development; Armacost \& Hosseini (1994) use hierarchical clustering to speed up decisions; Kwong \& Bai (2002) use this type of hierarchy to prioritize customer needs in an AHP-Fuzzy application; Scholl et. al. (2005) use incomplete hierarchy on an optimal university selection problem; Bañuls \& Salmeron (2008) use it to predict key areas in the information technology industry; Salgado et. al. (2012) adopts it to prioritize activities of a new product development model proposed for capital goods industry. Due to clustering (or, similarly, elimination of non-applicable judgments), this type of hierarchy requires only nine pairwise judgments for the incomplete hierarchy shown on Figure 2.

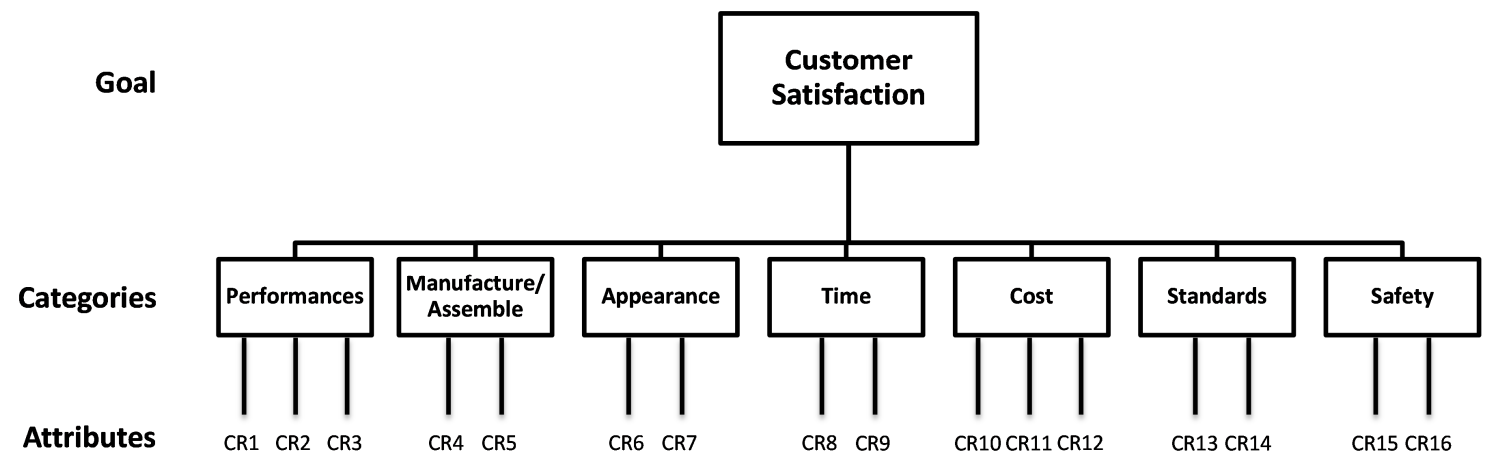

Figure 4. Incomplete hierarchy for customer needs prioritization (Kwong \& Bai, 2002). 


\section{Mathematical modeling}

Let us consider that, for the prioritization of $m$ elements, one collect judgments from $n$ expert individuals. For every individual it can be obtained a normalized vector of individual priorities, $\mathbf{w}=\left[w_{i j}\right]$, where $i$ refers to $i$-th individual and $j$ refers to the $j$-th element Since these vectors are normalized, they all satisfied Equation 1.

$$
\sum_{j=1}^{n} w_{i j}=1 \quad, \text { for } i=1,2, \ldots n
$$

In AIP, one can obtain the final priority vector either with the arithmetical mean, $\mathbf{a}=\left[a_{j}\right]$, (Equation 2$)$ or geometrical mean, $\mathbf{g}=\left[g_{j}\right]$, (Equation 3).

$$
\begin{array}{r}
a_{j}=\frac{\sum_{i=1}^{n} w_{i j}}{m} \\
g_{j}=\frac{\prod_{i=1}^{n} w_{i j}}{m}
\end{array}
$$

The aggregation by either arithmetic or geometric mean do not violate the Pareto Principle. However, the geometric mean was considered more consistent with the meaning of both judgments and priorities of AHP (Forman \& Peniwati, 1998). Recently, only the aggregation by geometric mean was described to get priorities from a crowd, combining judgments from people with different perspectives (Saaty, 2012). But, it was also observed that, with geometric mean, components of the final priority vector may not sum equal to one, needing an additional normalization.

As a matter of fact, the final vector will only sum equal to one in the rare case of unanimity. That is, $\sum_{i=1}^{n} g_{j}=1$ only if $w_{1 j}=w_{2 j}=\ldots=w_{2 j}$. In this case, $a_{j}=g_{j}$, for $j=1,2, \ldots m$.

Let us now consider the following very simple group decision-making example. Figure 5 presents an incomplete hierarchy, with four attributes (A1, A2, A3 and A4) grouped in two categories (C1 and C2). The decision goal is to prioritize the attributes. That is, to find out which is the most important attribute. 


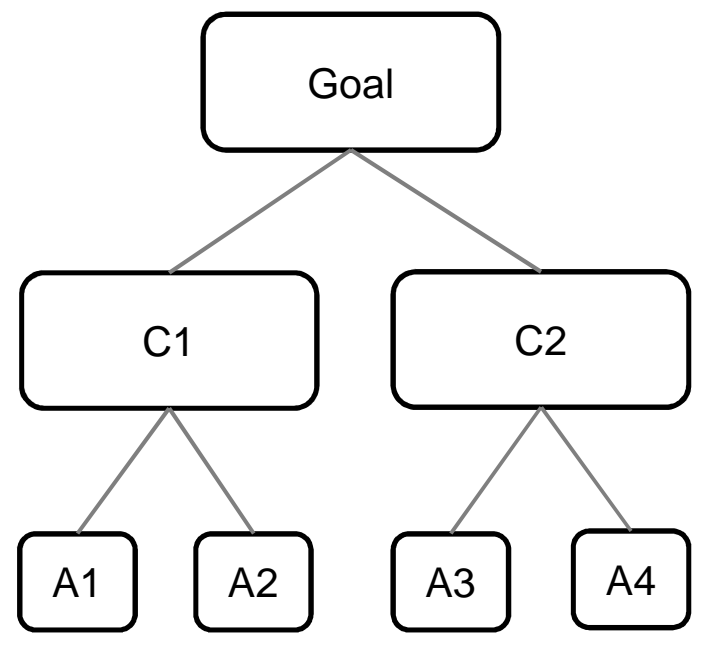

Figure 5. Example hierarchy with four attributes.

Tables 1a to $1 \mathrm{c}$ and $2 \mathrm{a}$ to $2 \mathrm{c}$ present judgment matrices and priorities from two individuals: $\mathrm{P} 1$ and $\mathrm{P} 2$, respectively.

Table 1a. Priorities for categories from P1.

\begin{tabular}{|l|l|l|l|}
\hline & $\mathrm{C} 1$ & $\mathrm{C} 2$ & Priority \\
\hline $\mathrm{C} 1$ & 1 & 2 & 0.667 \\
\hline $\mathrm{C} 2$ & $1 / 2$ & 1 & 0.333 \\
\hline
\end{tabular}

Table 1b. Priorities for attributes A1 and A2 from P1.

\begin{tabular}{|l|l|l|l|}
\hline & A1 & A2 & Priority \\
\hline A1 & 1 & 2 & 0.667 \\
\hline A2 & $1 / 2$ & 1 & 0.333 \\
\hline
\end{tabular}

Table 1c. Priorities for attributes A3 and A4 from P1.

\begin{tabular}{|l|l|l|l|}
\hline & A3 & A4 & Priority \\
\hline A3 & 1 & 3 & 0.750 \\
\hline A4 & $1 / 3$ & 1 & 0.250 \\
\hline
\end{tabular}

Table 2a. Priorities for categories from P2.

\begin{tabular}{|l|l|l|l|}
\hline & C1 & C2 & Priority \\
\hline C1 & 1 & 4 & 0.800 \\
\hline C2 & $1 / 4$ & 1 & 0.200 \\
\hline
\end{tabular}


Table 2b. Priorities for attributes A1 and A2 from P2.

\begin{tabular}{|l|l|l|l|}
\hline & A1 & A2 & Priority \\
\hline A1 & 1 & 5 & 0.833 \\
\hline A2 & $1 / 5$ & 1 & 0.167 \\
\hline
\end{tabular}

Table 2c. Priorities for attributes A3 and A4 from P2.

\begin{tabular}{|l|l|l|l|}
\hline & A3 & A4 & Priority \\
\hline A3 & 1 & $1 / 2$ & 0.333 \\
\hline A4 & 2 & 1 & 0.667 \\
\hline
\end{tabular}

Table 2 presents the aggregate priorities for categories using geometric and arithmetic means.

Table 2. Aggregate priority for categories by arithmetic and geometric means.

\begin{tabular}{|l|l|l|l|}
\hline & Arithmetic mean & Geometric mean & Normalized geometric mean \\
\hline C1 & 0.733 & 0.730 & 0.739 \\
\hline C2 & 0.267 & 0.258 & 0.261 \\
\hline Sum & 1 & 0.988 & 1 \\
\hline
\end{tabular}

Table 3 presents the local and overall priority for each attribute. It can be seen that, with arithmetic mean, priorities for attributes are already normalized, and they sum equal to priorities of categories. However, with the geometric mean priorities for attributes sum less than the priorities of categories. Priorities for $\mathrm{A} 1$ and $\mathrm{A} 2$, for instance, sum 0.754 against 0.739 of priority for $\mathrm{C} 1$.

Table 3. Aggregate priority for attributes by arithmetic and geometric means.

\begin{tabular}{|l|l|l|l|l|l|l|}
\hline & \multicolumn{2}{|l|}{ Arithmetic mean } & \multicolumn{2}{l|}{ Geometric mean } & \multicolumn{2}{l|}{ Normalized geometric mean } \\
\cline { 2 - 7 } & Local & Overall & Local & Overall & Local & Overall \\
\hline A1 & 0.750 & 0.550 & 0.745 & 0.544 & 0.759 & 0.573 \\
\hline A2 & 0.250 & 0.183 & 0.236 & 0.172 & 0.241 & 0.181 \\
\hline A3 & 0.542 & 0.145 & 0.500 & 0.129 & 0.551 & 0.136 \\
\hline A4 & 0.458 & 0.122 & 0.408 & 0.105 & 0.449 & 0.111 \\
\hline Sum & 2 & 1 & 1.889 & 0.950 & 2 & 1 \\
\hline
\end{tabular}

These results show that when aggregating individual priorities, geometric mean is not suitable to represent the overall priority for incomplete hierarchies. Three major problems became clear in this example:

- Sum of attributes' overall priorities is not equal to one;

- Sum of categories' priorities is not equal to one;

- Sum of attributes' overall priorities within a category is not equal to the category's priority.

In contrast, arithmetic mean gives normalized overall and local priorities directly from calculation and it gives correct results for priorities. None of the three problems occurs when using arithmetic mean. 
Normalizing geometric mean results after AIP does not solve all three problems detected. So, problems between overall and local priorities will be always present in real-world applications when geometric mean was used.

\section{Conclusions}

Mathematical modeling results showed in this paper that only arithmetic mean aggregation of individual priorities is suitable to be used when incomplete hierarchy is considered. This finding applies only to this described AHP scenario, where the incomplete hierarchy makes geometric mean not suitable for AIP because categories' and sub-categories' local priority aggregation do not comply with both normalization and sum of child alternatives' priorities. Normalization is very easy to achieve but it still does not solve the geometric mean problem. This finding may not impact on AHP applications with complete hierarchies or AIJ group decision-making.

This paper helps decision-makers to properly aggregate individuals' priorities, when considering an incomplete AHP hierarchy, to avoid problems among category's and its attributes' priorities. Several practical applications fall into this special situation, in particular, product development and service quality assurance surveys. In product development, for instance, AHP is being widely used to prioritize customers' needs and customers' requirements and these attributes are often grouped into functional and logical clusters. In this case, surveys take place considering a set of customers taken randomly from product's audience, which meets the special situation condition: incomplete AHP hierarchy and group decision comprising of separated individuals. As showed in this paper, using arithmetic mean to AIP in this situation is the only way to achieve correct group decision prioritization.

\section{REFERENCES}

Altuzarra, A., Moreno-Jimenez, J. M., \& Salvador, M. (2007). A Bayesian priorization procedure for AHP-group decision making. European Journal of Operational Research, 182, 367-382.

Armacost, R. L., Componation, P. J., Mullens, M. A., \& Swart, W. W. (1994). An AHP framework for prioritizing customer requirements in QFD: an industrialized housing application. IIE Transactions, 26, $72-79$.

Armacost, R. L., \& Hosseini, J. C. (1994). Identification of determinant attributes using the analytic hierarchy process. Journal of the Academy of Marketing Science, 22, 383-392.

Aykroyd, T. N. (2008). Value assessment of new product innovations. Massachusetts Institute of Technology.

Bañuls, V. A., \& Salmeron, J. L. (2008). Foresighting key areas in the Information Technology industry. Technovation, 28, 103-111.

Chan, L.-K., \& Wu, M.-L. (2005). A systematic approach to quality function deployment with a full illustrative example. Omega, 33, 119-139.

Davies, M. (2001). Adaptive AHP: a review of marketing applications with extensions. European Journal of Marketing, 35, 872-894. 


\section{K. de Souza Carmo, F. A. Silva Marins, V. A. P. Salomon, C. H. Pereira Mello/ Aggregation of individual priorities}

Forman, E., \& Peniwati, K. (1998). Aggregating individual judgments and priorities with the analytic hierarchy process. European Journal of Operational Research, 108, 165-169.

Kwong, C. K., \& Bai, H. (2002). A fuzzy AHP approach to the determination of importance weights of customer requirements in quality function deployment. Journal of Intelligent Manufacturing, 13, 367377.

Saaty, T. L. (1990). How to make a decision: The analytic hierarchy process. European Journal of Operational Research, 48, 9-26.

Saaty, T. L. (2006). Fundamentals of decision making and priority theory: with the analytic hierarchy process. Pittsburgh: RWS Publications.

Saaty, T. L. (2012). Getting Priorities from a Crowd: combining judgments from people with differing perspectives. International Journal of the Analytic Hierarchy Process, 4, 161-162.

Salgado, E. G., Salomon, V. A. P., \& Mello, C. H. P. (2012). Analytic hierarchy prioritisation of new product development activities for electronics manufacturing. International Journal of Production Research, 50, 4860-4866.

Scholl, A., Manthey, L., Helm, R., \& Steiner, M. (2005). Solving multiattribute design problems with analytic hierarchy process and conjoint analysis: An empirical comparison. European Journal of Operational Research, 164, 760-777.

Vaidya, O. S., \& Kumar, S. (2006). Analytic hierarchy process: An overview of applications. European Journal of Operational Research, 169, 1-29. 